\title{
Complications of Tissue Expansion
}

\author{
MARC D. BROWN, MD \\ TIMOTHY M. JOHNSON, MD
}

$\mathbf{T}$ he previous articles in this issue have dealt in depth with the technique of tissue expansion and its unique and remarkable advantages for soft tissue reconstruction. However, as with all aspects of cutaneous surgery, tissue expansion does have its share of disadvantages and potential complications (Table 1). The disadvantages of tissue expansion are relatively few. The procedure does require at least two operations and multiple visits to the physician are required during the 6 to 8 weeks of expansion. Up to 20 sterile injections may be required. ${ }^{1}$ Some authors have advocated inflation in the home setting, but this is not typically recommended. Tissue expansion may necessitate time away from work or school dependent in part upon how well the patient tolerates the cosmetic deformity and body image distortion that occurs with the procedure. Indeed, patient selection is extremely important prior to undertaking tissue expansion. The patient needs to be well informed, motivated, mentally competent, and fully understand the nature of the procedure. Patients invariably will respond to the obvious distortion in body image in a number of ways with negative reactions ranging from depression, to withdrawal, to even exhibitionist behavior. Some patients may simply be unable to tolerate tissue expansion because of unacceptable body image distortion and/or psychologic instability. Children can be an additional challenge although most are able to continue with their normal activities, including school. Successful expansion in the pediatric population is widely reported.

Complications of tissue expansion are more numerous and varied than are the simple disadvantages. Fortunately, most complications are minor and can be resolved without interruption of the expansion procedure. Reported rates of complications vary widely from 5 to $60 \% .^{2-5}$ The discrepancy of this rate is multifactorial, owing in part to the experience and expertise of the surgeon, the anatomic site of the expanded tissue, the health

From the Mohs Surgery Unit, Department of Dermatology, University of Rochester, Rochester, New York, and the Department of Dermatology, University of Michigan, Ann Arbor, Michigan.

Address correspondence and reprint requests to: Marc D. Brown, MD, Department of Dermatology, University of Rochester, 2180 South Clinton Avenue, Rochester, NY 14618. of the tissue undergoing expansion, and the degree of difficulty of the particular surgical case. Tissue expansion is not for the novice. For every surgical procedure, there is a learning curve, and this is very true for tissue expansion procedures. The cutaneous surgeon undertaking controlled tissue expansion should be well versed in soft tissue reconstruction and understand well the anatomy and physiology of the area being expanded. Those surgeons most experienced with this procedure initially reported complication rates of 25 to $40 \%$, but with time and experience these rates have dropped to 3 to $7 \%{ }^{4}$ This decline in the rate of complications is owing in part to the tremendous increase in understanding and judgment concerning tissue expansion, and also because the procedure of tissue expansion is no longer being reserved solely for the complicated and complex reconstructive cases but also for the relatively straightforward scar revision, scalp reduction, and breast reconstruction.

Anatomic site is a factor in the rate of complications. ${ }^{2,3}$ Complications are lower for trunk reconstructions, especially the breast. Expansion of the lower extremities appears to have a higher rate of complication. Even with intraoperative tissue expansion, complications are more significant for the lower extremities. ${ }^{6}$ Expansion that occurs in compromised skin and soft tissue (previously irradiated, burned, or scar tissue) not surprisingly shows higher rates of complications. This is most likely due to compromised vascularity.

Complications from controlled tissue expansion are listed in Table 1 and outlined below:

\section{Infection}

The rate of infection has been reported as high as $10 \%$ but in fact is relatively uncommon, occurring in about $1 \%$ of cases. ${ }^{7}$ The aim should be a sepsis rate of less than $0.5 \%{ }^{1}$ Perioperative antibiotics are recommended but prolonged antibiotic use is not. ${ }^{2}$ Irrigation with an antibiotic solution is recommended at the time of placement. If an expander does become infected, drainage, irrigation and antibiotic therapy is instituted immediately. If this is unsuccessful, the implant should be removed promptly. After the infection has completely subsided re-insertion may be possible. 
Table 1. Complications of Tissue Expansion
1. Infection
2. Implant extrusion
3. Mechanical failure
4. Hematoma/seroma
5. Pain
6. Nerve dysfunction
7. Tissue necrosis
8. Bone resorption
9. Flap failure

\section{Implant Extrusion}

Exposure of the implant usually occurs through necrosis of the overlying skin or through the original incision line. This complication commonly arises from an implant pocket created too small which subsequently places the implant in contact with the suture line. The pocket made by the surgeon at the initial procedure should be large enough to prevent the implant from being in immediate contact with the suture line. Too rapid expansion can also lead to excessive thinning, necrosis and subsequent extrusion. Prevention of implant extrusion is minimized by the following: 1) inflation of the expander should not be instituted for at least 1 to 2 weeks after placement of the implant; 2) the expander should be initially inflated only enough to fill the dead space; 3 ) expansion should proceed slowly; and 4) closure should be achieved in two layers with absorbable synthetic sutures used for the deeper layer. Secure steri strips or dressings should be used at the suture line after skin sutures are removed. Exposure of the implant requires antibiotic coverage but expansion can at times be continued with frequent inspection for infection. If the decision is made to continue expansion the patient should be placed on antibiotics until expansion is fully completed. If implant extrusion through an erosion site becomes more significant the expander should be removed and the procedure abandoned.

\section{Mechanical Failure}

Implant failure is a rare complication and overall the devices are highly reliable. Physician error is often the source of expander failure which includes breakage of the connecting tube, perforation of the envelope with the needle, and faulty assembly. ${ }^{1}$ Perforation will result in a slow leak but implant leakage does not always necessitate replacement and may not even be clinically apparent. Sterile saline is used as the injected fluid and thus a small leakage is a minor and usually insignificant complication. However, implant deflation requires replacement of the device with resumption of expansion after the incision has matured for one to two weeks. Puncture of the expander can be avoided by placing the reservoir at an appropriate distance from the expander. Silicone tubing at times will tear when stretched. Likewise, tube occlusion from kinking may occur.

\section{Hematoma}

Bleeding into the created pocket can be problematic. Fortunately it is uncommon. The risk of hematoma can be minimized by meticulous hemostasis at the time of implant insertion and the use of drains when necessary. Drains should be removed as soon as possible to prevent infection. When the hematoma occurs treatment is by prompt drainage and irrigation of the implant pocket. Antibiotic coverage should also be instituted. Prompt treatment of a hematoma can help to prevent both infection and increased tension on the expanded tissue.

\section{Seroma}

As the envelope of the expander is forced against the capsule following injection of saline, any fluid outside the expander in the potential space will be under pressure. Serum may then drain slowly through the needle hole over the injection port. It can be confused with a system leak unless a colored dye has been introduced into the expander. ${ }^{1}$ Larger accumulations of serosanguinous fluid can result in a seroma. This may require drainage or aspiration and should be closely followed for subsequent infection. Seroma formation is a relatively uncommon complication.

\section{Pain}

Most persons tolerate expansion well although it is uncomfortable. Pain may limit expansion but not commonly. The pain is due to tenseness of the expanded skin that normally occurs as the limit of expansion. Within 4 to 6 hours after expansion the discomfort is markedly abated and the patient is usually quite comfortable by 12

Figure 1. Central necrosis over forehead expanders.

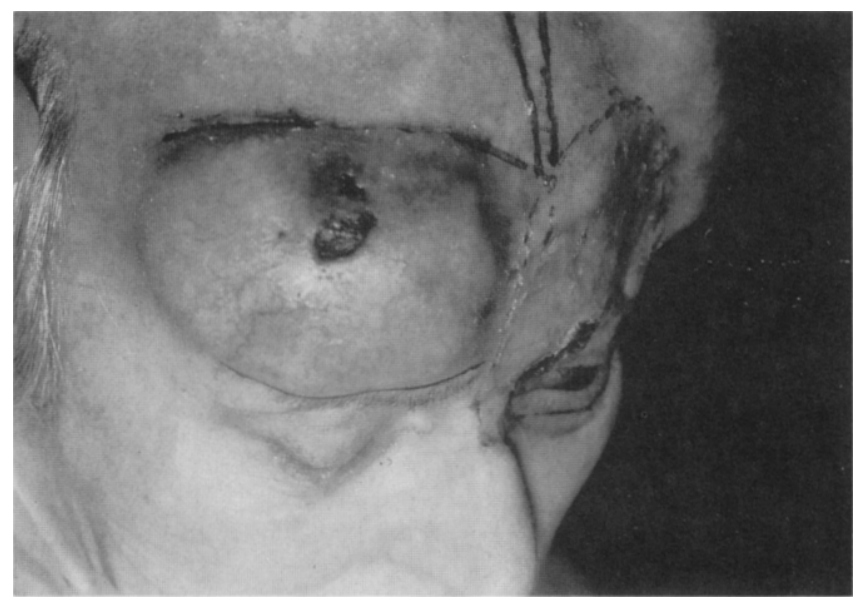


hours postexpansion. The degree of pain does correlate with anatomic site. There is minimal pain with expansion of breast tissue. Flank and back can be more painful; forehead, posterior occiput, and distal extremities (especially the foot) appear to cause the most pain. ${ }^{1,3,5}$

\section{Tissue Necrosis}

Overzealous inflation of the expander can compress the overlying tissue such that it becomes ischemic (Figure 1). In most instances, pain will prevent this. Persistent pain and/or blanching of the surrounding tissue should alert the physician to this potential complication. A small amount of saline should be withdrawn to allow for capillary refill. Skin that is previously burned, scarred, or irradiated is more prone to tissue necrosis. Pressure dressings need to be secure and firm but not excessively tight over the newly expanded skin in order not to compromise vascularity. Folding of the silicone expander beneath a thin flap can result in local pressure necrosis. A rigid backing plate on a convex surface can lead to local pressure damage and ischemia. A poorly placed injection port such as that overlying bone, can result in skin breakdown.

\section{Nerve Dysfunction}

Neuropraxia is a rare complication despite expansion procedures with the implant placed over major nerves. Nerve dysfunction may result from compression due to direct mechanical force or traction on a nerve because of anatomic restriction. Neuropraxia usually resolves rapidly if recognized promptly and treated before the onset of focal demyelination.

\section{Bone Resorption}

Calvarial bone resorption has been described in forehead reconstruction. ${ }^{8}$ Overall, bone resorption is a very rare complication. Pediatric patients should be watched more closely for this occurrence.

\section{Flap Failure}

In most cases expansion creates a highly viable flap. However, flap failure has been described, usually reflect- ing unfavorable tissue conditions or too rapid implant inflation. Partial flap necrosis has been observed even after a perfectly normal expansion process.

In summary, complications of tissue expansion do occur and patients need to be advised of such preoperatively. Infection, implant exposure, and implant failure may interrupt the intended reconstruction but can be properly managed and still allow for completion of the procedure. With experience and proper precautions the total complication rate for tissue expansion should be less than $10 \%$, with major complications $5 \%$ or less. Complications will continue to decrease with increased experience and knowledge of the use of tissue expansion. However, even in the most experienced and skilled surgical hands, complications (both seen and unforeseen) will occur; early recognition and management becomes imperative.

\section{References}

1. Masser MR. Tissue expansion: a reconstructive revolution or a cornucopia of complications. Br J Plast Surg 1990;43: 244-8.

2. Argenta LC, Marks MW, Pasyk KA. Advances in tissue expansion. Clin Plast Surg 1988;12:159-71.

3. Antonyshyn O, Gruss JS, MacKinnon SE, et al. Complications of soft tissue expansion. Br J Plast Surg 1985;41: $239-49$.

4. Austad ED. Complications in tissue expansion. Clinics in Plastic Surgery 1987;3:549-50.

5. Manders EK, Schenden MJ, Furrey JA, et al. Soft tissue expansion: concepts and complications. Plast Reconstr Surg $1984 ; 74: 493-507$.

6. Sasaki GH. Intraoperative sustained limited expansion (ISLE) as an immediate reconstructive technique. Clin Plast Surg 1987;14:563-73.

7. Marans J, Horan DB, Robinson JK. Tissue expansion: past, present and future. J Am Acad Dermatol 1990;23:813-25.

8. Fudern GM, Orgel MG. Full thickness erosion of the skull secondary to tissue expansion in the pediatric burn patient. Plast Reconstr Surg 1988;82:840-8. 